\title{
Injection Performance of a Gas-Solid Injector Based on the Particle Trajectory Model
}

\author{
Daolong Yang, ${ }^{1}$ Jianping Li, ${ }^{1}$ Changlong Du, ${ }^{1}$ Hongxiang Jiang, ${ }^{1}$ and Kehong Zheng ${ }^{1,2}$ \\ ${ }^{1}$ College of Mechanical and Electrical Engineering, China University of Mining and Technology, Xuzhou 221116, China \\ ${ }^{2}$ College of Mines and Earth Sciences, University of Utah, Salt Lake City, UT 84112-0114, USA \\ Correspondence should be addressed to Jianping Li; lijp1021@126.com
}

Received 26 March 2015; Accepted 20 April 2015

Academic Editor: Luigi Nicolais

Copyright (c) 2015 Daolong Yang et al. This is an open access article distributed under the Creative Commons Attribution License, which permits unrestricted use, distribution, and reproduction in any medium, provided the original work is properly cited.

\begin{abstract}
Gas-solid injectors are widely used feeding equipment in pneumatic conveying systems. The performance of a gas-solid injector has a significant influence on the type of application it can be employed for. To determine the key factors influencing the injection performance and address clogging problems in a gas-solid injector during a pneumatic conveying process, the particle trajectory model has been utilised as a means to perform simulations. In the particle trajectory model, the gas phase is treated as a continuous medium and the particle phase is treated as a dispersed phase. In this work, numerical and experimental studies were conducted for different nozzle positions in a gas-solid injector. A gas-solid injector test-bed was constructed based on the results of the simulations. The results show that the nozzle position is the key factor that affects the injection performance. The number of extrusive particles first increases and then decreases with the change in the nozzle position from left to right. Additionally, there is an optimum nozzle position that maximises the injection mass and minimises the number of particles remaining in the hopper. Based on the results of this work, the injection performance can be significantly increased and the clogging issues are effectively eliminated.
\end{abstract}

\section{Introduction}

A gas-solid injector is an important piece of feeding equipment in pneumatic conveyers, which are widely used in the petroleum and chemical industries, material conveying, power stations, and other departments. They possess a simple structure, no moving parts, and concatenate conveniently with other pieces of equipment $[1,2]$. Many methods have been used to simulate gas-solid fluidisation. Some examples include the two-fluid smoothed particle hydrodynamics method (TF-SPH) [3], the multilattice deterministic trajectory model (MLDT) [4], the multifluid model (MFM), the two-fluid model (TFM) [5], and the discrete phase model (DPM) [6]. In addition, particle trajectory models (PTMs) are also widely used because they require fewer partial differential equations when addressing the dispersed phase and because they provide exact results when forecasting the particle distribution [7-9]. The particle trajectory model is mainly used to forecast the movement of dilute particle phases in a turbulent flow. The Lagrangian solution model is adopted to address the particle phase. The particle-particle and particle-wall collisions are calculated using a statistical method that is not restricted by the number of particles $[10,11]$.

Practically, gas-solid injectors may jam depending on the particle diameter and as the volume being conveyed increases. These issues restrict their application and development when dealing with large-sized particles and largemass flow pneumatic conveying. Many scholars have studied the conveying properties and static pressure distributions in gas-solid injectors and agree that the location of the driving nozzle and the angle of the converging section have an obvious influence on the maximum achievable mass flow rate [12-15]. The goal of this paper is to improve the injection performance of a gas-solid injection (to increase the conveying properties) and determine the key factors that affect the injection performance. The particle trajectories were simulated in a uniform flow field using the PTM and the injection performance at different driving nozzle locations was obtained by analysing the number of injected particles. Finally, a gas-solid injector test-bed was built to investigate 
the injection performance and verify the simulation models and results.

\section{Mathematical Model}

The movement of the particles is determined by the interactions between the solid and gas phases, which are determined in two ways. The first is by the exchange of mass, momentum, and energy. The second is through particle-particle and the particle-wall collisions. The particle trajectories are obtained by the PTM, which uses different handling methods for the gas and particle phases. The gas phase is treated as a continuous medium and the variables of the gas phase are obtained by solving the gas control equation. The particle phase is treated as a dispersed phase and the Lagrangian method is used to track the particle trajectories. The mass, momentum, and energy of the gas and particle phases are exchanged during the iterative process [16].

2.1. Gas Control Equation. The gas phase is treated as a continuous medium in the PTM. Therefore, the gas phase continuity and momentum equations are based on the law of conservation of mass and Newton's second law [17, 18]. In addition, the interactions between the gas and particles are considered. For larger particle diameters of up to $5 \mathrm{~mm}$, additional interactions are added to the gas control continuity equation:

$$
\begin{aligned}
\frac{\partial \rho}{\partial t}+\frac{\partial}{\partial x_{j}}\left(\rho v_{j}\right) & =S, \\
S & =-n_{p} m_{p},
\end{aligned}
$$

where $\rho$ is the density of gas phase, $x_{j}$ is the coordinate of $j$ direction, $v_{j}$ is the velocity component of the gas phase in the $x_{j}$ direction, $S$ is the particle phase volume fraction in the gas-solid two-phase mixture, $n_{p}$ is the number density of the particle phase, and $m_{p}$ is the single particle mass. The momentum equation is defined as

$$
\begin{aligned}
\frac{\partial}{\partial t}\left(\rho v_{i}\right)+\frac{\partial}{\partial x_{j}}\left(\rho v_{j} v_{i}\right) & \\
= & -\frac{\partial p}{\partial x_{i}}+\Delta \rho g_{i}+v_{i} S+\frac{\partial}{\partial x_{j}}\left[\mu\left(\frac{\partial v_{j}}{\partial x_{i}}+\frac{\partial v_{i}}{\partial x_{j}}\right)\right] \\
& +\frac{\sum \rho_{p}\left(v_{p i}-v_{i}\right)}{\tau_{r}}
\end{aligned}
$$

where $\mu$ is the dynamic viscosity of the gas phase, $x_{i}$ is the coordinate of $i$ direction, $v_{i}$ is the velocity component of the gas phase in the $x_{i}$ direction, $\rho_{p}$ is the density of the particle phase in the gas-solid two-phase mixture, $v_{p i}$ is the velocity component of gas phase in the $x_{i}$ direction, and $\tau_{r}$ is the diffuse relaxation time of the particle phase. Equations (1) and (2) are obtained from $[17,18]$.

The above control equations are all for the gas phase. There are a total of four control equations when we consider the momentum equations and the three directions in

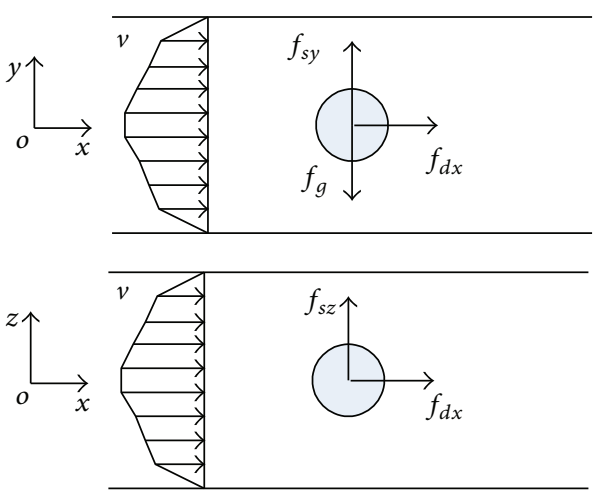

FIGURE 1: Forces and motion of particle in horizontal uniform flow.

the coordinate system. Therefore, the unknown variables for the gas phase are $\rho, v_{i}, v_{j}, v_{p k}$, and $T$. These are addressed by solving the closed form of the $k-\varepsilon$ turbulence model [19]. The turbulent kinetic energy and turbulent momentum dissipation equations are shown respectively:

$$
\begin{aligned}
\frac{\partial}{\partial x_{i}}\left(\rho k u_{i}\right)= & \frac{\partial}{\partial x_{j}}\left[\left(\mu+\frac{\mu_{t}}{\sigma_{k}}\right) \frac{\partial k}{\partial x_{j}}\right]+G_{k}+G_{b}-\rho \varepsilon \\
& -Y_{M}+S_{k}, \\
\frac{\partial}{\partial x_{i}}\left(\rho \varepsilon u_{i}\right)= & \frac{\partial}{\partial x_{j}}\left[\left(\mu+\frac{\mu_{t}}{\sigma_{\varepsilon}}\right) \frac{\partial \varepsilon}{\partial x_{j}}\right] \\
& +C_{1 \varepsilon} \frac{\varepsilon}{k}\left(G_{k}+C_{3 \varepsilon} G_{b}\right)-C_{2 \varepsilon} \rho \frac{\varepsilon^{2}}{k}+S_{\varepsilon} .
\end{aligned}
$$

Equations (3) and (4) are obtained from Yakhot and Orszag's work [20].

2.2. Trajectory Equation of Particle Phase. The force analysis on the particle phase was performed using Newton's second law in the PTM and the particle trajectory equations were acquired by integrating twice.

2.2.1. Motion Equation. The particle motion equation (Figure 1) is shown in the following equation, which is obtained from Newton's second law:

$$
m_{p} \frac{d v_{p}}{d t}=f_{d}+f_{g}+f_{F},
$$

where $v_{p}$ is the particle velocity, $m_{p}$ is the average particle mass, $f_{d}$ is the fluid drag force on the particle, $f_{g}$ is the force of gravity on the particle, and $f_{F}$ represents the other forces on the particle.

The other particle forces include the Saffman force, the Brown force, and the Magnus force. In this study, the particle diameter is $5 \mathrm{~mm}$, meaning that the Brown force and the Magnus force have little effect on the particle movement, as described in [21-24]. Therefore, these forces are ignored when considering the particle state of motion. 
The fluid drag force is defined as [25]

$$
f_{d}=\frac{3}{4} \frac{\rho m_{p}}{\rho_{p} d_{p}} c_{D}\left(v-v_{p}\right)\left|v-v_{p}\right|,
$$

where $\rho_{p}$ is the particle density, $d_{p}$ is the particle diameter, $c_{D}$ is the fluid drag coefficient, which is defined as $c_{D}=0.44$ when the particle diameter is $5 \mathrm{~mm}, v$ is the gas velocity, and $v_{p}$ is the particle velocity.

The Saffman force is defined as

$$
f_{S}=\frac{2 K v^{1 / 2} \rho d_{i j}}{\rho_{p} d_{p}\left(d_{l k} d_{k l}\right)^{1 / 4}}\left(v-v_{p}\right),
$$

where $K$ is the Saffman force constant, which is defined as $K=2.594$ and $d_{i j}$ is the deformation tenser.

The Saffman force is generated by the different velocity gradients in the fluid. Equation (7) is obtained from $\mathrm{Li}$ and Ahmadi [25], which determines the succession of the Saffman expression [26].

2.2.2. Particle Trajectory. When the particles are in a uniform flow field and do not collide with the walls or other particles, as described in (5), the particle trajectory equations may be defined as follows:

$x$-direction:

$$
m_{p} \frac{d^{2} x_{p}}{d t^{2}}=f_{d x}
$$

$y$-direction:

$$
m_{p} \frac{d^{2} y_{p}}{d t^{2}}=f_{d y}+f_{g}+f_{S y}
$$

$z$-direction:

$$
m_{p} \frac{d^{2} z_{p}}{d t^{2}}=f_{d z}+f_{S z}
$$

assuming that the initial particle velocities in the $x$ - and $y$ directions are zero. By substituting (6) and (7) into (8) (10), the particle phase trajectory equations in the $x$-, $y$-, and $z$ directions may be obtained by integrating twice:

$x$-direction:

$$
x_{p}=x_{p 0}+v_{x} t-\frac{1}{a} \ln \left(a v_{r x} t+1\right),
$$

$y$-direction:

$$
\begin{aligned}
y_{p}= & y_{p 0}+\left(v_{y}+\frac{b}{2 a}-\frac{c}{\sqrt{a}}\right) t \\
& +\frac{1}{c} \sqrt{\frac{f}{a}} \ln \frac{(f-1) e^{2 c t}}{e^{2 c t} f-1},
\end{aligned}
$$

$z$-direction:

$$
z_{p}=z_{p 0}+v_{z} t-\sqrt{\frac{n}{a}} \ln \frac{n e^{b t / \sqrt{a}}-1}{n e^{b t / \sqrt{a}}}
$$

where $x_{p 0}, y_{p 0}$, and $z_{p 0}$ are the initial particle coordinates in the $x$-, $y$-, and $z$-directions, $a$ is the computational coefficient of fluid drag, $b$ is the computational coefficient of the Saffman force, $c$ is the coefficient of the simplified equation, $v_{x}, v_{y}$, and $v_{z}$ are the gas velocities in the $x-, y$-, and $z$-directions, $v_{r}$ is the difference in the initial gas and particle velocities, and $f$ and $n$ are the coefficients generated when simplifying the integral computation. These coefficients are shown in

$$
\begin{aligned}
a & =\frac{4 \rho_{p} d_{p}}{3 \rho c_{D}}, \\
b & =\frac{2 K v^{1 / 2} \rho d_{i j}}{m_{p} \rho_{p} d_{p}\left(d_{l k} d_{k l}\right)^{1 / 4}}, \\
c & =\frac{a g}{1+a b}, \\
v_{r i} & =v_{i}-v_{p i 0} \quad(i=x, y, z) \\
f & =\frac{2 a v_{r i}-b-\sqrt{b^{2} \pm 4 a g}}{2 a v_{r i}-b+\sqrt{b^{2} \pm 4 a g}} \\
n & =\frac{a v_{r i}-b}{a v_{r i}} .
\end{aligned}
$$

The particle velocity recovery factor for the collisions with the wall is determined from Alister's experiment [27]. There is a close relationship between the recovery factor and the particle impact angle, which is defined as

$$
\begin{aligned}
e_{n}= & 0.988-0.78 \theta+0.19 \theta^{2}-0.024 \theta^{3}+0.027 \theta^{4}, \\
e_{r}= & 1-0.78 \theta+0.84 \theta^{2}-0.21 \theta^{3}+0.028 \theta^{4} \\
& -0.022 \theta^{5}
\end{aligned}
$$

where $e_{n}$ is the normal recovery factor, $e_{r}$ is the tangential recovery factor, and $\theta$ is the particle impact angle.

\section{Simulations}

The flow field of the gas phase in the gas-solid injector is calculated using a colocated grid with the SIMPLE method. The particle coordinate positions are solved using the equations of motion. Additionally, the solid volume percentage of the colocated grids is also calculated. Then, the calculated value is returned for the calculation of the flow field. In this method, the coupled solution for the determination of the continuous fluid and particle phases may be alternated. 

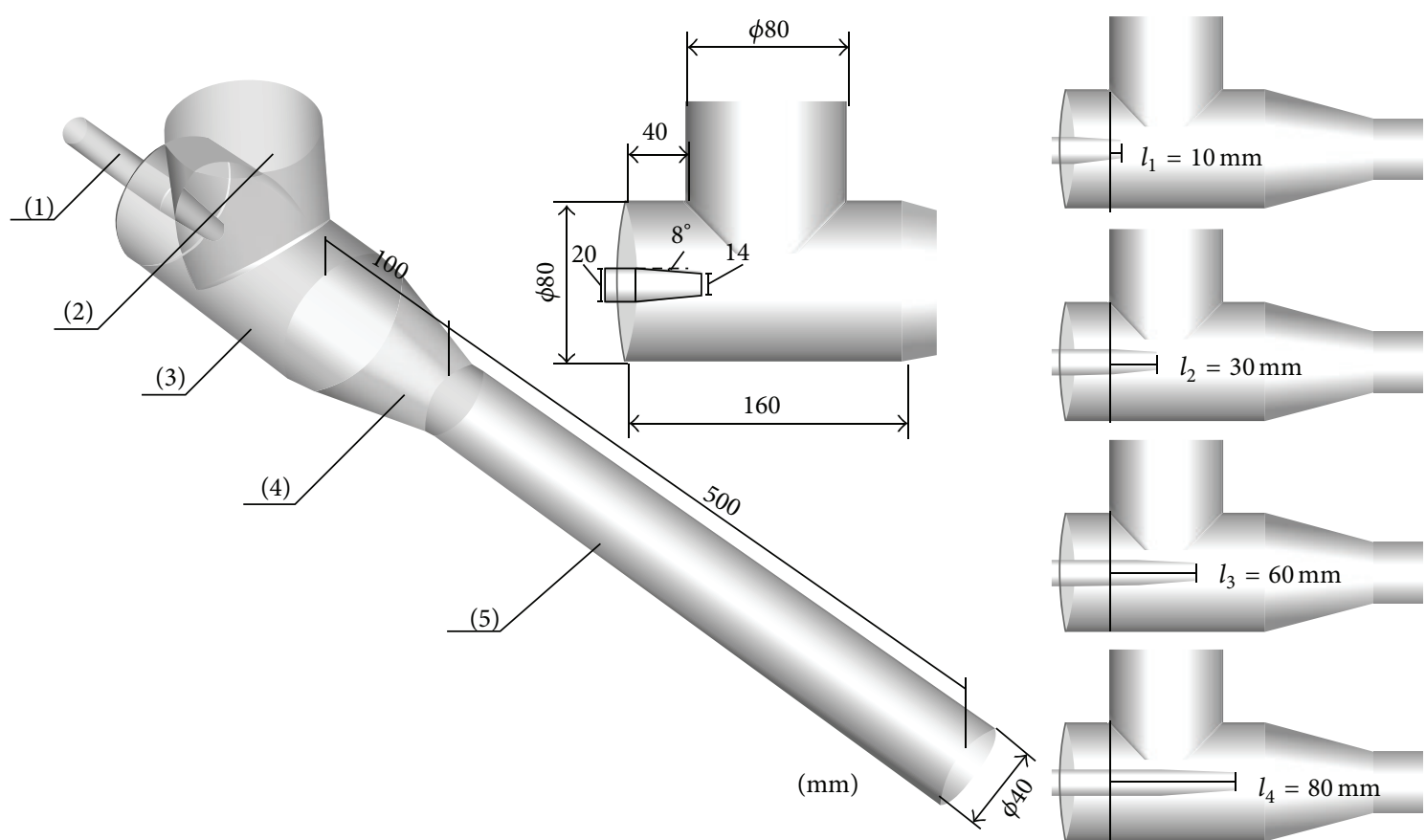
(1) Driving nozzle
(4) Contraction section
(2) Feed opening
(5) Delivery pipe

(3) Mixing room

FIGURE 2: Simulation model of injector.

TABLE 1: Initial parameters of simulation model.

\begin{tabular}{|c|c|c|c|c|c|}
\hline \multicolumn{2}{|l|}{ Injector } & \multicolumn{2}{|c|}{ Gas phase } & \multicolumn{2}{|c|}{ Particle phase } \\
\hline \multirow{4}{*}{ Nozzle location } & $l_{1}=0 \mathrm{~mm}$ & Density & $\rho=1.205 \mathrm{~kg} / \mathrm{m}^{3}$ & Diameter & $d=1 \sim 5 \mathrm{~mm}$ \\
\hline & $l_{2}=30 \mathrm{~mm}$ & Viscosity & $\mu=1.81 \times 10^{-5} \mathrm{~kg} * \mathrm{~s} / \mathrm{m}^{2}$ & Velocity & $v_{p y}=-4.50 \mathrm{~m} / \mathrm{s}$ \\
\hline & $l_{3}=60 \mathrm{~mm}$ & Kinematic viscosity & $v=1.512 \times 10^{-5} \mathrm{~m}^{2} / \mathrm{s}$ & Density & $\rho=2719 \mathrm{~kg} / \mathrm{m}^{3}$ \\
\hline & $l_{4}=80 \mathrm{~mm}$ & Temperature & $T=20^{\circ} \mathrm{C}$ & Feeding time & $T_{1}=0.20 \mathrm{~s}$ \\
\hline Diameter of feed opening & $D_{r}=80 \mathrm{~mm}$ & Humidness & $20 \%$ & & $T_{2}=0.40 \mathrm{~s}$ \\
\hline Diameter of delivery pipe & $D_{c}=45 \mathrm{~mm}$ & Working pressure & $P=0.50 \mathrm{MPa}$ & Mass flow & $Q=0.50 \mathrm{~kg} / \mathrm{s}$ \\
\hline
\end{tabular}

3.1. Simulation Model and Initial Parameters. The gas-solid injector consists of a driving nozzle, feed opening, mixing chamber, contraction section, and delivery pipe (Figure 2). The inner diameter of the driving nozzle is $20 \mathrm{~mm}$, the diameter of the delivery pipe is $14 \mathrm{~mm}$, and the inclination angle is $8^{\circ}$. The nozzle locations addressed by the four models are $l_{1}=10 \mathrm{~mm}, l_{2}=30 \mathrm{~mm}, l_{3}=60 \mathrm{~mm}$, and $l_{4}=80 \mathrm{~mm}$, respectively. The initial parameters for the simulation model are shown in Table 1. The feeding time was set to be $0.2 \mathrm{~s}$ and $0.4 \mathrm{~s}$, respectively, to prevent the material from becoming plugged in the gas-solid injector.

3.2. Simulation Results. One group of steady-state simulations and two groups of transient simulations were conducted for different feeding times and particle trajectories in the gassolid injector to analyse the injection mass.

The coordinates at each time step are solved using the PTM. The particle trajectories are shown in Figure 3 after the particle coordinates are projected into the gas-solid injection model. The nozzle locations at $l_{1}=10 \mathrm{~mm}, l_{2}=30 \mathrm{~mm}$, $l_{3}=60 \mathrm{~mm}$, and $l_{4}=80 \mathrm{~mm}$ are shown in Figure $3-(1),(2)$, (3), and (4), respectively.

The injection block not only increases the use cost but also reduces the reliability of the pneumatic conveying system. Reducing the number of particles in the injector may alleviate material blockage issues and achieve a balance between the feeding mass and the extrusive mass. A statistical analysis of the extrusive number and the residual number of particles is conducted separately for each of the four models. The statistical results are shown in Figures 4-7.

The number of extrusive particles and particles remaining in the hopper for a $0.2 \mathrm{~s}$ feeding time is shown in Figures 4 and 5, respectively, where the horizontal axis represents the simulation time and the vertical axis represents the number of particles remaining in the hopper.

The number of extrusive particles and the number of particles remaining in the hopper for a $0.4 \mathrm{~s}$ feeding time are shown in Figures 6 and 7, respectively, where the horizontal 


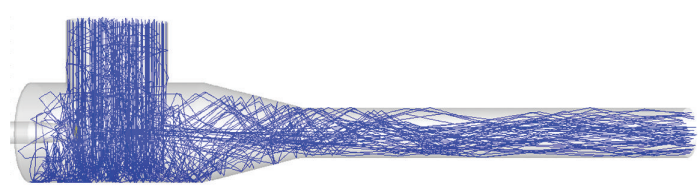

(1) Nozzle location $l_{1}=10 \mathrm{~mm}$

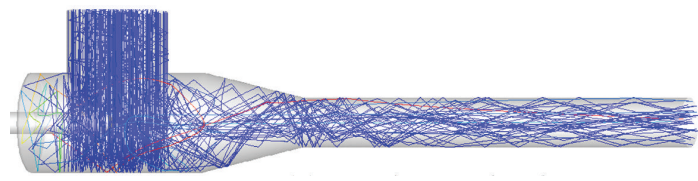

(2) Nozzle location $l_{2}=30 \mathrm{~mm}$

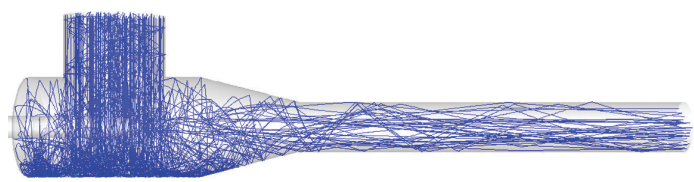

(3) Nozzle location $l_{3}=60 \mathrm{~mm}$

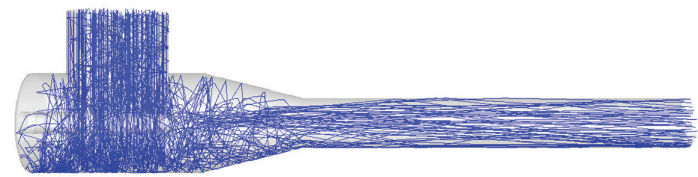

(4) Nozzle location $l_{4}=80 \mathrm{~mm}$

Figure 3: Part of particle trajectories.

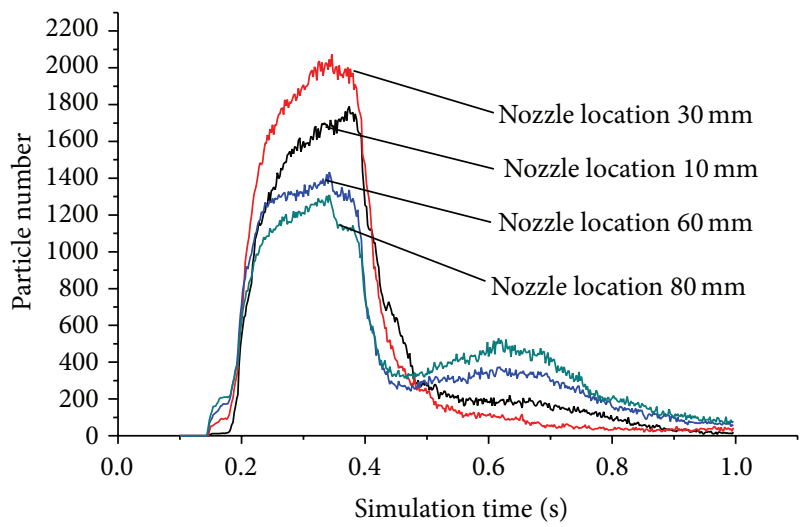

FIgURE 4: The number of extrusive particles in $0.2 \mathrm{~s}$ feeding time.

axis represents the simulation time and the vertical axis represents the remaining number of particles in the hopper.

\section{Experiment}

4.1. Experimental Equipment. The gas-solid injector test-bed consists of a gas inlet, a bolt, flange, support frame, injector, and hopper (Figure 8). One side of the gas inlet connects the gas-holder and the other side inserts into the injector through the flange and is fixed by the bolt, which can move up and down in the axial direction.

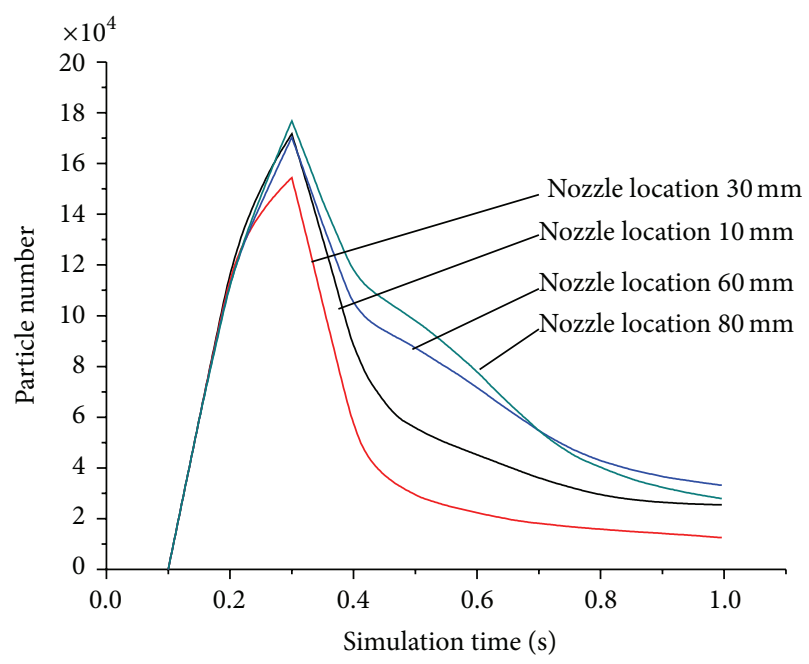

FIgURE 5: The number of remaining particles in $0.2 \mathrm{~s}$ feeding time.

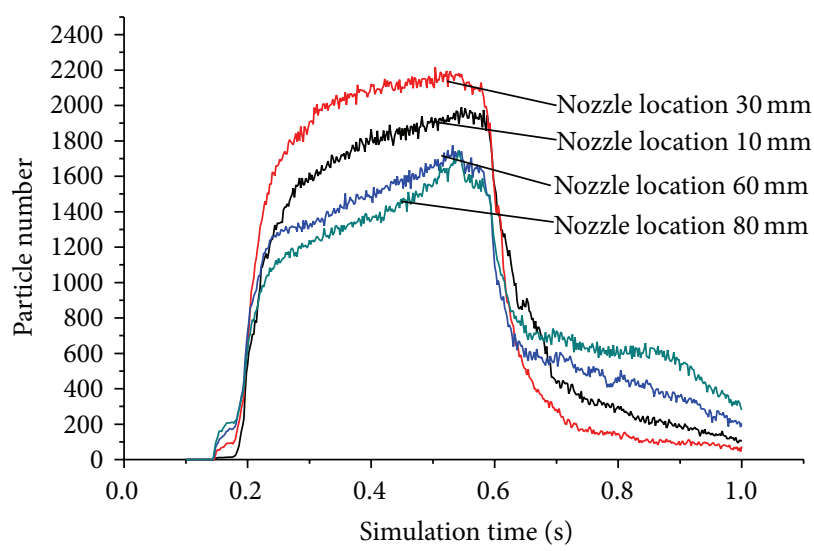

Figure 6: The number of extrusive particles in $0.4 \mathrm{~s}$ feeding time.

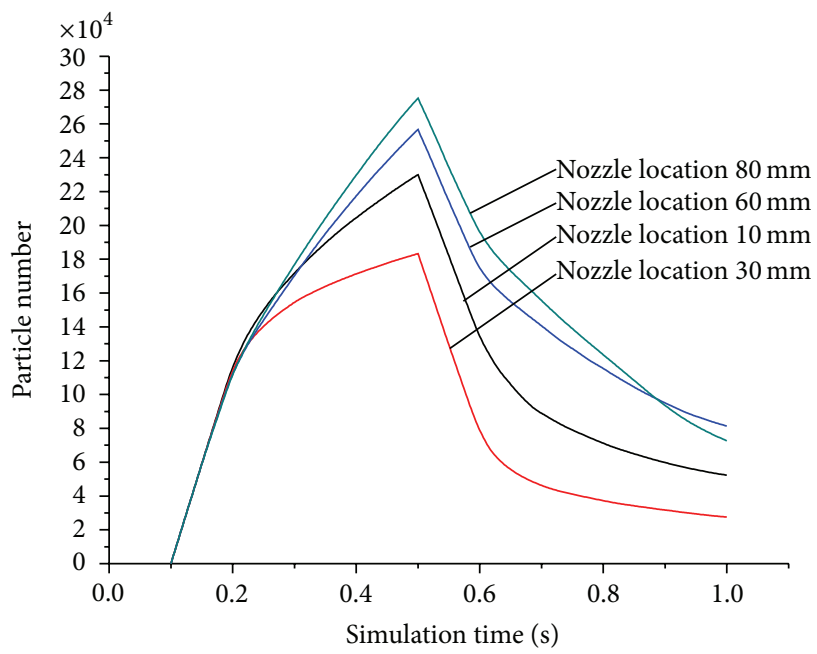

FIGURE 7: The number of remaining particles in $0.4 \mathrm{~s}$ feeding time. 
TABLE 2: Injection mass of gas-solid injector test-bed.

\begin{tabular}{|c|c|c|c|c|c|c|c|}
\hline \multirow{2}{*}{ Nozzle location } & \multicolumn{7}{|c|}{ Injection mass $/\left(\mathrm{m}^{3} / \mathrm{h}\right)$} \\
\hline & 1 & 2 & 3 & 4 & 5 & 6 & Average \\
\hline A & 0.08 & 0.10 & - & - & - & - & - \\
\hline B & 0.96 & 1.16 & 1.09 & 0.95 & 0.91 & 1.08 & 1.030 \\
\hline $\mathrm{C}$ & 2.85 & 2.90 & 2.63 & 2.56 & 2.84 & 2.65 & 2.743 \\
\hline $\mathrm{D}$ & 3.52 & 3.49 & 3.58 & 3.45 & 3.66 & 3.20 & 3.497 \\
\hline $\mathrm{E}$ & 2.74 & 2.61 & 2.84 & 3.02 & 2.91 & 2.61 & 2.790 \\
\hline $\mathrm{F}$ & 2.45 & 2.31 & 2.12 & 2.16 & 2.35 & 2.51 & 2.321 \\
\hline G & 2.05 & 1.90 & 2.31 & 2.28 & 1.95 & 2.12 & 2.095 \\
\hline $\mathrm{H}$ & 0.63 & 0.54 & 0.64 & 0.82 & 0.72 & 0.54 & 0.648 \\
\hline I & 0.21 & 0.15 & 0.22 & 0.08 & 0.15 & 0.21 & 0.170 \\
\hline
\end{tabular}

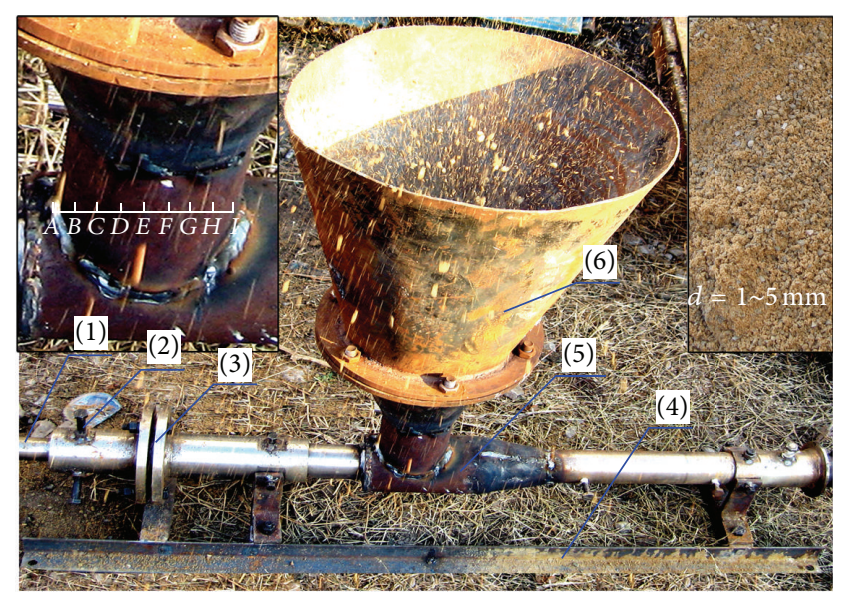
(1) Gas inlet
(4) Support frame
(2) Bolt
(5) Injector
(3) Flange
(6) Hopper

FIGURE 8: Gas-solid injector test-bed.

4.2. Test Objective and Method. A mixture of sediment and stone was used with the gas-solid injector test-bed to explore the influence of the nozzle position on the injection performance and verify the simulation results. The material supply pipe had an inner diameter of $80 \mathrm{~mm}$ and is divided into eight equal parts along the axial direction of mixture chamber. These parts are denoted by A to I from left to right. The nozzle was placed in a position and the mixture was continually dropped into the hopper. The injected material was gathered every five minutes after a relatively stable injection mass is achieved. The results from the tests are shown in Table 2.

4.3. Experiment Results. The jet gas flow caused a significant number of impacts and there was a great deal of noise when the nozzle was located at point $\mathrm{A}$. The material erupted in reverse from the hopper, leading to the failure of the test. Only a handful of the material was injected into the mixture chamber because the material could not be mixed with the high-speed gas when the nozzle was located at point I. The average injection mass was adjusted by eliminating the data at

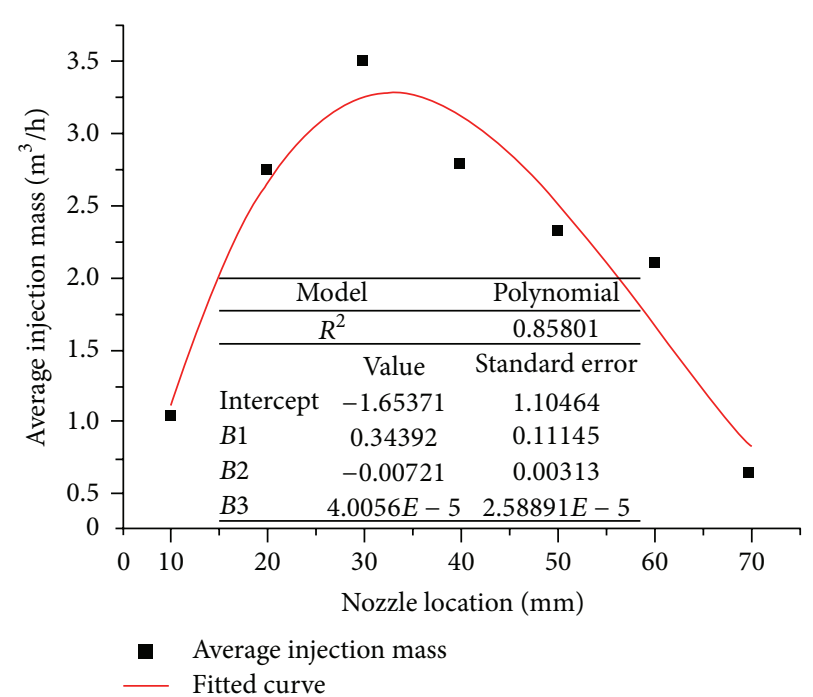

FIGURE 9: Relation between nozzle's location and average injection mass.

nozzle locations A and I. The fitted curve is shown in Figure 9 and the relationship between the average injection mass and the nozzle location is expressed in

$$
y=-1.6571+0.34392 x-7.21 \times 10^{-3} x^{2}
$$

which achieved an $R$-squared value of $R^{2}=0.85801$.

\section{Discussion}

The particle trajectories in the mixing chamber of the gassolid injectors, which are shown in Figure 3-(1), are sparser compared to the other three models when using the PTM, which indicates less particle deposition and particle-wall collisions. In contrast, the particle trajectories shown in Figures 3-(3) and 3-(4) are denser, indicating more particle deposition and energy consumption, an unstable flow field, and a greater degree of wear in mixing chamber. The particle trajectories in the delivery pipe, shown in Figure 3-(4), exhibit a greater degree of linearity compared to the other three models when using the PTM, which indicates less collisions 
between the particles and wall. The other three models exhibit clear particle-particle or particle-wall collisions. In general, a greater number of collisions lead to a greater degree of wear and higher energy consumption in the pipes. As a result, the material conveying process becomes more difficult.

The particles are injected over $0.15 \mathrm{~s}$ and the number of extrusive particles increases from $0.18 \mathrm{~s}$ to $0.22 \mathrm{~s}$. From $0.25 \mathrm{~s}$ to $0.34 \mathrm{~s}$, the particle count increases steadily before beginning a downward trend after $0.35 \mathrm{~s}$ and sharply declines during the $0.38 \mathrm{~s} \sim 0.50 \mathrm{~s}$ period (Figure 4 ). The number of extrusive particles is largest when the nozzle is at $l_{2}=30 \mathrm{~mm}$. Additionally, a small increase occurs from $0.50 \mathrm{~s}$ to $0.75 \mathrm{~s}$ when the nozzle is at $l_{3}=60 \mathrm{~mm}$ and $l_{4}=80 \mathrm{~mm}$. This illustrates that, during this time, the sedimentary particles at the bottom of injector reenter the flow field and are ejected out of the mixing chamber.

The number of remaining particles is less using the PTM than that for the other three injector models when the nozzle location is at $l_{2}=30 \mathrm{~mm}$ after $0.22 \mathrm{~s}$ (Figure 5). Remarkably, the number of particles for all four models peaks at $0.30 \mathrm{~s}$ (after the feeding stops). The curves are similar for nozzle locations at $l_{2}=30 \mathrm{~mm}$ and $l_{1}=10 \mathrm{~mm}$ after $0.30 \mathrm{~s}$. The curves are also similar when the nozzle location is at $l_{3}=$ $60 \mathrm{~mm}$ and $l_{4}=80 \mathrm{~mm}$. However, the number of particles remaining in the hopper in the latter is less than the former after $0.70 \mathrm{~s}$ to the end of the injection period because the flow field experienced acute fluctuations when the sedimentary particles quickly reentered the flow field.

As the feeding time increases, the number of extrusive particles reaches a maximum when the nozzle location is at $l_{2}=30 \mathrm{~mm}$ (Figure 6). The number of extrusive particles increases steadily from $0.25 \mathrm{~s}$ to $0.50 \mathrm{~s}$ but increases slowly when the nozzle position is at $l_{2}=30 \mathrm{~mm}$, indicating that the feeding and injecting masses are balanced. The number of extrusive particles tends to decline after $0.55 \mathrm{~s}$ and then declines sharply from $0.58 \mathrm{~s}$ to $0.62 \mathrm{~s}$. This is the same trend observed in Figure 3 for a nozzle position of $l_{4}=80 \mathrm{~mm}$. The number of extrusive particles increases slightly after $0.70 \mathrm{~s}$. However, this does not appear when the nozzle position is $l_{3}=60 \mathrm{~mm}$. The number of remaining particles in the four models is clearly different from each other; the number of remaining particles in the PTM is less than that for the other three models for a nozzle position of $l_{2}=30 \mathrm{~mm}$ (Figure 7). After $0.5 \mathrm{~s}$, the material feed stops and the number of extrusive particles linearly decreases in all of the models.

The simulation results show that the number of extrusive particles is maximised when the nozzle is positioned at $l_{1}=30 \mathrm{~mm}$, indicating that the injection performance is very good and the number of remaining particles is then minimised. However, the particle-wall collisions are a significant issue in the delivery pipe. The linearity of particle trajectories is better and fewer particle-wall collisions are observed compared to that for the other three models for a nozzle location of $l_{4}=80 \mathrm{~mm}$. However, the particle trajectories are complex and the number of remaining particles is maximised. Consequently, a nozzle location of $l_{2}=30 \mathrm{~mm}$ is chosen for the gas-solid injector test-bed to verify the simulation results regarding the injection performance.
The experimental results show that nozzle location is the key factor that influences the injection performance, especially the injection mass. The injection mass increases first and then decreases as the nozzle position moves from left to right (or from A to I), indicating that there is an optimum nozzle position and thus verifying the simulation.

When the nozzle was in position $\mathrm{A}$, the jet gas flow caused serious impacts, leading to significant noise and resulted in little mass being ejected. The material erupted in reverse from the hopper and blocked the mixing chamber during continuous feeding.

When the nozzle was in position $\mathrm{D}$, the injection mass was maximised and the average maximum injection mass was $3.497 \mathrm{~m}^{3} / \mathrm{h}$ with a peak injection mass of $3.66 \mathrm{~m}^{3} / \mathrm{h}$. These injection masses were determined to have met the requirements for the engineering application.

When the nozzle was in position I, the injection air did not mix with the material. Only a small amount of material was ejected out the mixing chamber and most of the material did not leave the hopper.

Due to the limitations of the experimental conditions, this paper only addresses the injection performance from the view of the nozzle position. There are other parameters that influence the injection properties of a gas-solid injector that are not examined here, such as the geometric construction of the mixing chamber, the wall conditions, and the material properties. Future studies are expected to examine these aspects.

\section{Conclusions}

Based on the theoretical analysis, the conclusions obtained from the simulation and experimental studies regarding key factors that affect the injector performance are as follows:

(1) The nozzle location is the key factor affecting the gassolid injector performance. There is an optimal nozzle location at which the number of extrusive particles is maximised and the number of particles remaining in the hopper is minimised.

(2) The simulation results indicate that injection performance peaks when the nozzle is positioned at $30 \mathrm{~mm}$. However, the particle-wall and particle-particle collisions are more intense in the delivery pipe at this nozzle position. The particle trajectories are more linear and the particle-wall collisions decrease for a nozzle position of $90 \mathrm{~mm}$. The particle trajectories at this nozzle position are more complicated, and the greatest amount of remaining particles in the hopper is observed for this nozzle position.

(3) The results of the experimental study indicate that the nozzle location has a clear influence on the injection performance. The injection performance improves remarkably in the optimum nozzle position of the gas-solid injector when the structural parameters do not change. 


\section{Conflict of Interests}

The authors declare that there is no conflict of interests regarding the publication of this paper.

\section{Acknowledgments}

This project is supported by National High-Tech Research and Development Program of China (863 Program) (no. 2012AA062102), the Graduate Student Innovation Training Project in Jiangsu Province (nos. KYLX_1379 and CXLX13_936), and the Priority Academic Program Development of Jiangsu Higher Education Institutions.

\section{References}

[1] W.-Y. Yu, R. Tian, and S.-Y. Yan, "Numerical simulation on the flow structure and performance of the ejector," Journal of Engineering Thermophysics, vol. 33, no. 11, pp. 1881-1883, 2012.

[2] H. J. Chen, W. Lu, C. Cao, and L. Yang, "Applicability analysis on ejectors with cylindrical and conical mixing chambers," Journal of Chemical Industry and Engineering, vol. 64, no. 6, pp. 20432049, 2013.

[3] L. J. Deng, Y. N. Liu, W. Wang, W. Ge, and J. H. Li, "A two-fluid smoothed particle hydrodynamics (TF-SPH) method for gassolid fluidization," Chemical Engineering Science, vol. 99, pp. 89101, 2013.

[4] H. Zhang, M. Liu, B. Wang, and X. Wang, "Dense gas-particle flow in vertical channel by multi-lattice trajectory model," Science in China Series E: Technological Sciences, vol. 55, no. 2, pp. 542-554, 2012.

[5] K. Hong, Z. Shi, W. Wang, and J. Li, "A structure-dependent multi-fluid model (SFM) for heterogeneous gas-solid flow," Chemical Engineering Science, vol. 99, pp. 191-202, 2013.

[6] K. Liu, J.-S. Bai, and P. Li, "Application of particle trajectory model in 1D planar ejection," Journal of Central South University of Technology, vol. 15, no. 1, pp. 149-154, 2008.

[7] M. A. I. Rasel, M. A. Taher, and H. D. Kim, "A study on the gas-solid particle flows in a needle-free drug delivery device," Journal of Thermal Science, vol. 22, no. 4, pp. 340-344, 2013.

[8] Z. G. Feng, E. E. Michaelides, and S. L. Mao, "Simulation of particle-wall collisions in a viscous fluid using a resolved discrete particle method," in Proceedings of the Asme Fluids Engineering Division Summer Conference, vol. 1, pp. 57-62, 2010.

[9] H. Sakaino, "A semitransparency-based optical-flow method with a point trajectory model for particle-like video," IEEE Transactions on Image Processing, vol. 21, no. 2, pp. 441-450, 2012.

[10] C. F. You, H. L. Zhao, Y. Cai, H. Y. Qi, and X. C. Xu, "Experimental investigation of interparticle collision rate in particulate flow," International Journal of Multiphase Flow, vol. 30, no. 9, pp. 1121-1138, 2004.

[11] Z. Z. An, H. Zhou, Y. Yang, and X. L. Shi, "A study on particle trajectory of particle swarm optimization," Applied Mechanics and Materials, vol. 433-435, pp. 662-666, 2013.

[12] Y.-Q. Xiong and M.-Y. Zhang, "Experimental study on static pressure distribution properties of gas-solid injector under pressure," Journal of Combustion Science and Technology, vol. 10, no. 2, pp. 130-134, 2004.

[13] Y.-Q. Xiong, M.-Y. Zhang, Z.-L. Yuan, and D.-M. Xue, “Threedimensional numerical simulation on gas-solid two-phase flows in gas-solid injector," Proceedings of the Chinese Society of Electrical Engineering, vol. 25, no. 20, pp. 77-82, 2005.

[14] Y. Q. Xiong, M. Y. Zhang, and Z. L. Yuan, “Three-dimensional numerical simulation method for gas-solid injector," Powder Technology, vol. 160, no. 3, pp. 180-189, 2005.

[15] Y. Q. Xiong, Z. L. Yuan, and M. Y. Zhang, “Three-dimensional numerical simulation on conveying properties of gas-solid injector under pressurization," Journal of Chemical Industry and Engineering, vol. 10, no. 55, pp. 1638-1643, 2004.

[16] A. Levy, "Two-fluid approach for plug flow simulations in horizontal pneumatic conveying," Powder Technology, vol. 112, no. 3, pp. 263-272, 2000.

[17] D. Z. Zhang and W. B. VanderHeyden, "The effects of mesoscale structures on the macroscopic momentum equations for twophase flows," International Journal of Multiphase Flow, vol. 28, no. 5, pp. 805-822, 2002.

[18] J. D. Franklin and J. S. Lee, "Momentum/continuity coupling with large non-isotropic momentum source terms," International Journal for Numerical Methods in Fluids, vol. 61, no. 9, pp. 946-969, 2009.

[19] A. Mehdizadeh, B. Firoozabadi, and S. A. Sherif, "Particle trajectory study in submerged flows with baffles using $\overline{v^{2}}-f$ and $k-\varepsilon$ turbulence models," Journal of Fluids Engineering, vol. 132, no. 5, Article ID 051105, 10 pages, 2010.

[20] V. Yakhot and S. A. Orszag, "Renormalization group analysis of turbulence. I. Basic theory," Journal of Scientific Computing, vol. 1, no. 1, pp. 3-51, 1986.

[21] N. Huber and M. Sommerfeld, "Modelling and numerical calculation of dilute-phase pneumatic conveying in pipe systems," Powder Technology, vol. 99, no. 1, pp. 90-101, 1998.

[22] Z. C. Zheng, X. Y. Zou, X. F. Yang, and H. Cheng, "Simulation of effects of the Saffman force and the Magnus force on sand saltation in turbulent flow," in Proceedings of the ASME International Mechanical Engineering Congress and Exposition (IMECE '11), vol. 6, pp. 501-506, Denver, Colo, USA, November 2011.

[23] Z. Zhu and J. Lin, "Pressure gradient force, saffman lift, and magnus lift on the fiber-like particle in fluid," Journal of Dong Hua University, vol. 17, no. 2, pp. 23-27, 2000.

[24] Q. Li, V. Rudolph, F. Y. Wang, and M. Horio, "A study of particle packing compression under fluid drag force by DEM simulations," Developments in Chemical Engineering and Mineral Processing, vol. 13, no. 5-6, pp. 693-708, 2005.

[25] A. Li and G. Ahmadi, "Dispersion and deposition of spherical particles from point sources in a turbulent channel flow," Aerosol Science and Technology, vol. 16, no. 4, pp. 209-226, 1992.

[26] P. G. Saffman, "The lift on a small sphere in a slow shear flow," Journal of Fluid Mechanics, vol. 22, no. 2, pp. 385-400, 1965.

[27] A. Forder, M. Thew, and D. Harrison, "A numerical investigation of solid particle erosion experienced within oilfield control valves," Wear, vol. 216, no. 2, pp. 184-193, 1998. 

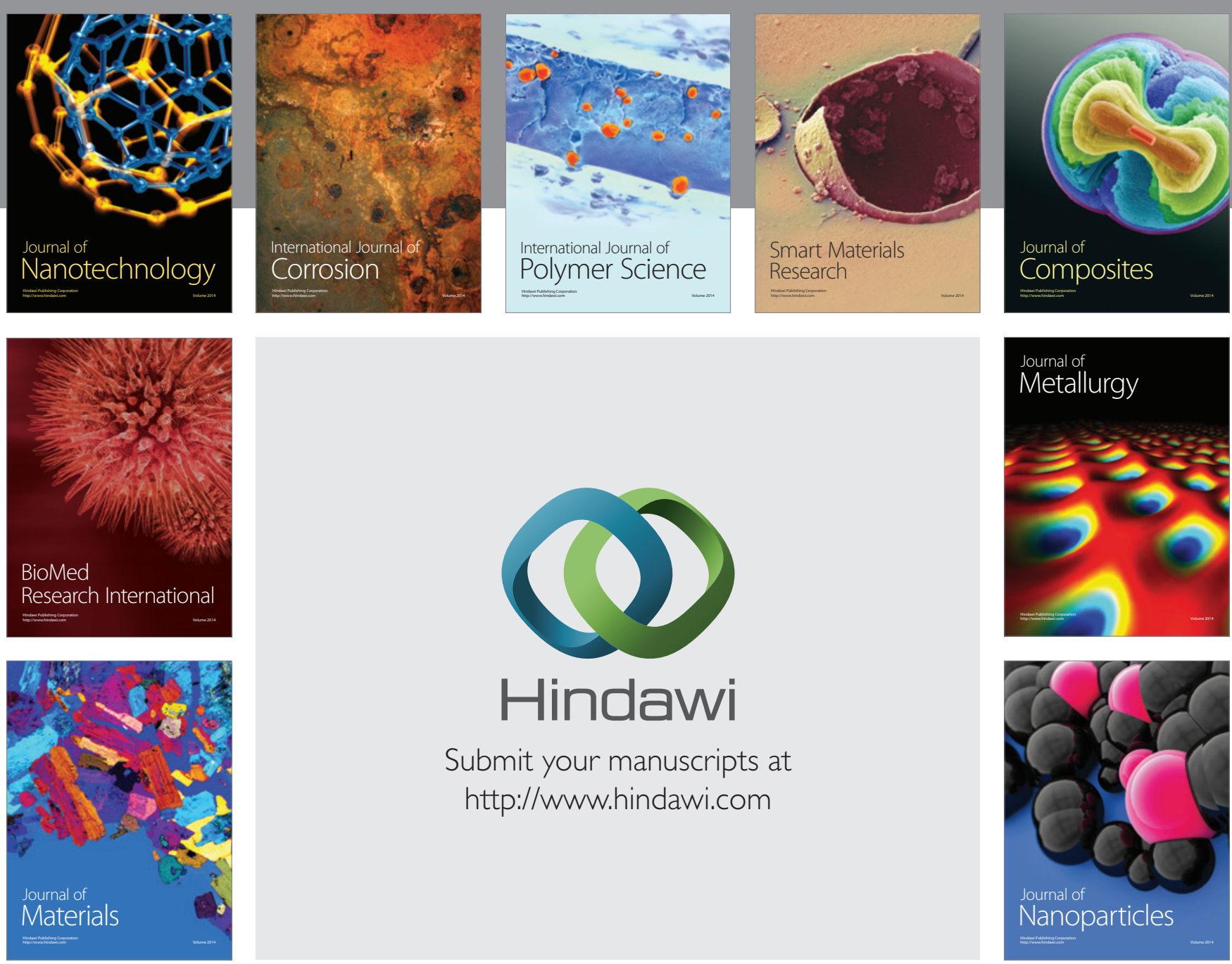

Submit your manuscripts at http://www.hindawi.com
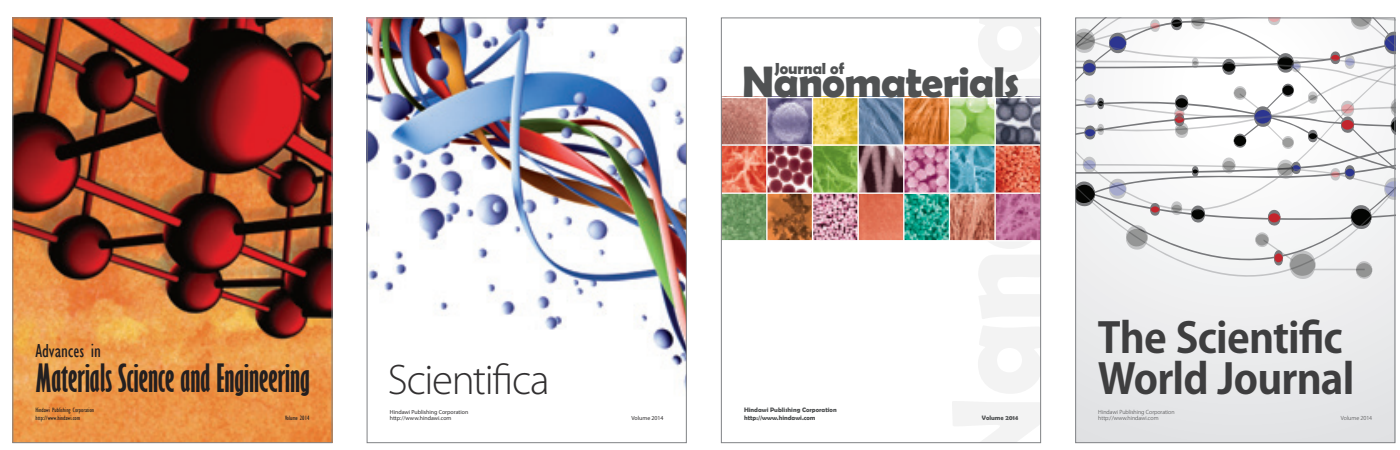

\section{The Scientific World Journal}
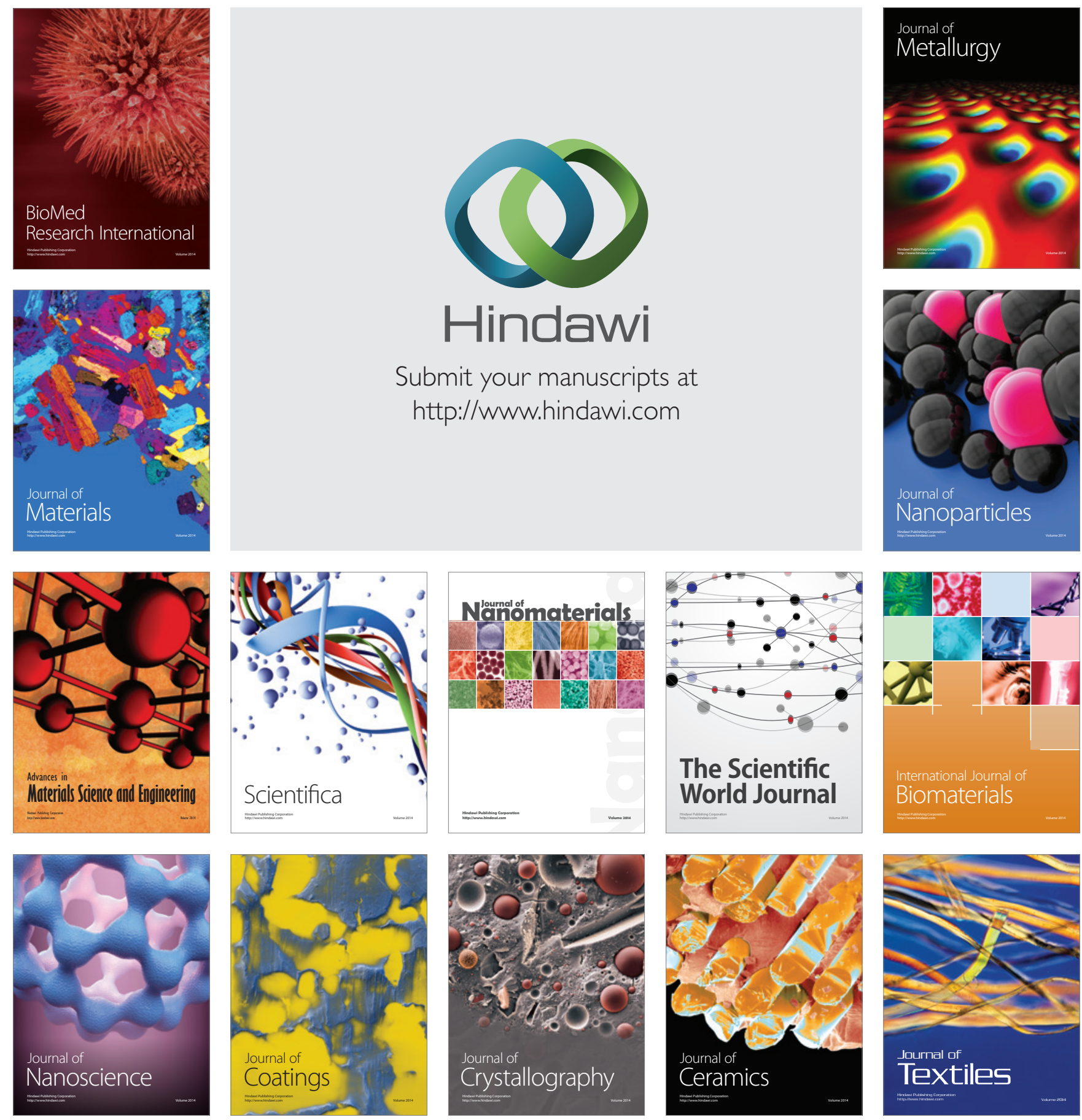